\title{
Monitoreo de variables meteorológicas a través de un sistema inalámbrico de adquisición de datos
}

Monitoring of meteorological variables through a wireless data acquisition system

\author{
Daniel Camilo Ruiz-Ayala' \\ Carlos Arturo Vides-Herrera ${ }^{2}$ \\ Aldo Pardo-García ${ }^{3}$
}

Recibido: octubre 24 de 2017

Aceptado: diciembre 28 de 2017

\section{Resumen}

En este artículo se presenta el desarrollo de un sistema para el monitoreo inalámbrico de variables climáticas. El diseño se realizó a partir de microcontroladores de Microchip, los cuales realizan la adquisición, almacenamiento y transmisión inalámbrica de las señales digitales. Igualmente, el microcontrolador emplea un reloj en tiempo real para saber la fecha y hora de adquisición de las muestras. El hardware también cuenta con cinco canales para la conexión de sensores y una memoria Micro SD para almacenamiento de información, junto con un módulo Wi-Fi para la supervisión inalámbrica de las variables. La información se sube a un servidor que aloja la página web, diseñada para visualizar los datos desde cualquier ordenador con conexión a internet. Adicionalmente, se desarrolló una aplicación Android que permite visualizar los datos desde dispositivos móviles con ese sistema operativo. El rendimiento del sistema fue satisfactorio, luego de comparar los datos adquiridos con los de una estación meteorológica comercial, que sirvió de patrón. Se concluye que los microcontroladores continúan siendo dispositivos adecuados para implementar sistemas de adquisición de datos, que al ser combinados con aplicativos desarrollados a la medida, brindan soluciones competitivas y a un costo razonable.

Palabras clave: adquisición de datos, monitoreo meteorológico, sistema inalámbrico.

\begin{abstract}
In this article we present the development of a system for the wireless monitoring of climatic variables. The design was made from microchip microcontrollers, which perform the acquisition, storage and wireless transmission of digital signals. Likewise, the microcontroller uses a clock in real time to know the date and time of acquisition of the samples. The hardware also has five channels for connecting sensors and a Micro SD memory for storage of data, along with a Wi-Fi module for wireless monitoring of variables. The information is uploaded to a server that hosts the web page, designed to view the data from any computer with an internet connection. Additionally, an Android application was developed that allows data to be viewed from mobile devices with that operating system. The performance of the system was satisfactory, after comparing the data acquired with those of two commercial meteorological stations, which served as a pattern. It is concluded that microcontrollers continue to be adequate devices to implement data acquisition systems, which when combined with customized applications, provide competitive solutions at a reasonable cost.
\end{abstract}

Keywords: data acquisition, meteorological monitoring, wireless system.

1 Ingeniero Electrónico, Contratista CENS, Pamplona, Colombia. E-mail: danielcamilor@gmail.com

2 Ingeniero Electrónico, Magíster en Controles Industriales, Universidad de Pamplona, Pamplona, Colombia. E-mail: carlosarturvi@ unipamplona.edu.co

3 Ingeniero Electricista con énfasis en electrónica, Doctor en Ciencias, Universidad de Pamplona, Pamplona, Colombia. E-mail: apardo13@hotmail.com 


\section{Introducción}

La medición de variables meteorológicas ha crecido en importancia ya que la información suministrada por las estaciones es vital para monitorear el cambio climático, el comportamiento de las cuencas hidrográficas y en la determinación de recursos eólicos y solares, así como en el establecimiento de políticas relacionadas con el medio ambiente (IDEAM, 2016; Pabón-Fernández, Díaz-Rodríguez \& Pardo-García, 2016; Serna-Mendoza, Vélez-Rojas \& Londoño-Pineda, 2016). El registro permanente de variables climáticas durante largos periodos de tiempo en zonas remotas o inhóspitas, sin contar con la presencia de un personal capacitado que se encargue de proporcionar datos presentables con el menor porcentaje de error, hace que aparezca la necesidad de automatizar las estaciones meteorológicas (Pardo-García \& Castellanos-González, 2017).

Uno de los dispositivos importantes dentro de una estación es el Datalogger, que se encarga de recoger la información de varios sensores meteorológicos (Márquez-Marín, 2004). En la industria existe diversidad de parámetros que caracterizan la fabricación de un Datalogger, como: tipo de medición, temperatura de trabajo, tasa de medición y almacenaje de la información, funciones especiales, entre otras; todo esto con el fin de que el usuario encuentre un instrumento que se adapte a sus necesidades (Altamirano-Santillán, Vallejo-Vallejo \& Cruz-Hurtado, 2017; Almario-Ospino, Ramón-Valencia \& Ramón-Valencia, 2011; OMM, 2010).

Al encontrar variedad de instrumentos surge la diversidad de precios en el mercado, donde la mayoría son de costos relativamente altos debido a su complejidad y funcionamiento (Cargua, Cueva, Escobar, Arciniegas \& Nieto-Guerrero, 2017). Adicionalmente, al encontrar instrumentos económicos estos carecen de la circuitería necesaria para satisfacer totalmente las necesidades de medición, almacenaje y monitoreo que el usuario pueda requerir (Vázquez, Toledo, Mason \& Canalí, 2005).

Hoy en día es posible diseñar este tipo de sistemas teniendo los conocimientos y bases de su funcionamiento (Moreno-Rubio, Jiménez-López \& Barrera-Lombana, 2013). Esto unido a que la tecnología de captación y procesamiento de señales ha avanzado, permitiendo la generación de sistemas complejos que se pueden adaptar a los instrumentos que emplea la meteorología para sus investigaciones (Sánchez-Dams, 2013; Figueroa-Cuello, Pardo-García \& Díaz-Rodríguez, 2017).

En el presente documento se describe el desarrollo de un sistema de adquisición, registro y monitoreo inalámbrico de variables climáticas. En la siguiente sección se ilustra la estructura del Datalogger, cuyo principal componente es un microcontrolador; además, se presentan los componentes y estructura del sistema de adquisición de datos, tanto en hardware como en software. Luego se describen los resultados de la implementación del sistema en condiciones reales y se brindan las conclusiones del estudio.

\section{Materiales y métodos}

\subsection{Diseño propuesto}

Se propone el desarrollo de un sistema de captación de las variables propias de las condiciones climáticas como: temperatura, humedad, radiación solar, dirección y velocidad del viento, entre otras. El equipo debe ajustarse a la operación en condiciones adversas, propias de los ambientes externos. También se requiere de un sistema de monitoreo en línea, que permita el acceso permanente y en tiempo real a las condiciones climatológicas de la ciudad donde opere la estación (Rodríguez-Jiménez, Agueda \& Portela-Lozano, 2004). En la figura 1 se presenta el diagrama de bloques del sistema propuesto. 


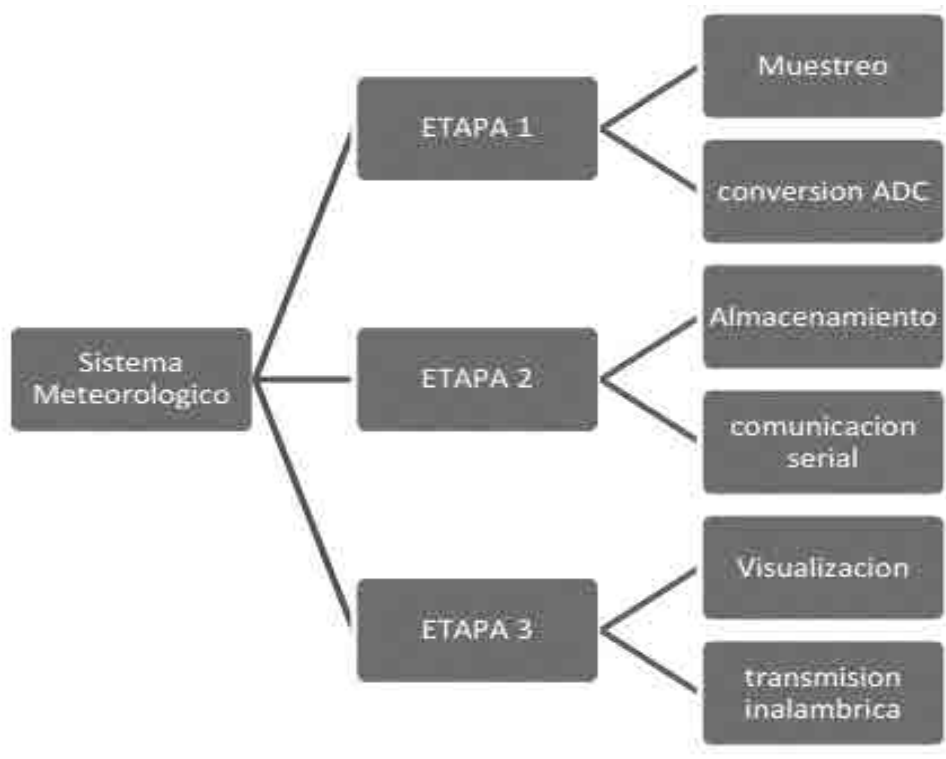

Figura 1. Etapas del sistema meteorológico propuesto.

El sistema se divide en dos etapas: la primera corresponde a los sensores que se conectan a un microcontrolador (Microchip, 2017), el cual se encarga de procesar la información de seis sensores, para obtener los datos de temperatura, humedad relativa, presión atmosférica, radiación solar o luminosidad, dirección y velocidad del viento. Esta etapa se forma por sensores con una respuesta digital y analógica, donde cada uno requiere de un circuito de instrumentación, y un código para adquirir y convertir las magnitudes climáticas en valores estándar que se puedan comparar con otros sistemas climáticos (Sandoval, Tobar-Molano, Mosquera \& González, 2011; Moreno-Anselmi, Reyes-Ortiz \& Ruíz-Acero, 2016).

El datalogger de la estación meteorológica se encarga de registrar la información en una memoria micro SD, generando un formato.XLS de MS-Excel. Cada muestra registrada lleva su hora y fecha, la cual es suministrada por un Reloj en Tiempo Real, RTC; además, el tiempo de muestreo varía desde 1 segundo hasta 4 minutos, dependiendo de la necesidad del usuario. Adicionalmente, se dispone de un software y una página web que, por medio de un servidor, permite acceder desde un dispo- sitivo móvil con sistema operativo Android, que también fue desarrollado.

A continuación se describen brevemente los dispositivos empleados en el diseño del sistema meteorológico para la monitorización de las variables climáticas.

Memoria SD: esta etapa contiene una micro SD, donde serán guardados los datos que luego son leídos por diversos programas para su análisis y visualización.

Módulo GPS: el GPS, Global Positioning System por sus siglas en inglés, transmite la información codificada en un protocolo denominado NMEA. Las tramas de información entregadas por el GPS están en formato texto, codificadas en ASCII. Para fines de diseño es importante identificar la velocidad de transmisión del módulo GPS y ajustarla en el módulo USART del microcontrolador (Vargas-Guativa, López-Velásquez \& Conde-Cárdenas, 2014).

Módulo WiFi: este módulo es el encargado de la transmisión inalámbrica del datalogger. Para po- 
ner en marcha el sistema, se deben enviar por la UART una serie de comandos básicos TCP / IP Toolbox AT, con los que se configura: velocidad de transmisión, conexiones a internet y modo de operar, entre otros. El módulo WiFi ESP8266, al conectarse a una red, recibe automáticamente una dirección IP dinámica, asignada por el router.

Software de desarrollo: en este proyecto se utilizaron dos software principales para el desarrollo de las aplicaciones: Visual Basic 2010 y Android Studio (Santiago \& Sánchez-Allende, 2016; Mercado-Ramos, Zapata \& Ceballos, 2015). El primero se utilizó para elaborar el software de captura de datos provenientes del Hardware, cuya información se guarda en una base de datos. El software desarrollado tiene la capacidad de desplegar todas las variables en tiempo real, permitiendo efectuar el análisis de datos de forma gráfica. El programa Android Studio sirvió para elaborar la APP orientada a dispositivos móviles.

Hosting y dominio de la página WEB: para alojar la página se requiere de un Hosting que cumpla los siguientes requerimientos: PHP MyAdmin, Base de Datos, MYSQL remota, Cpanel, que acepte programación de páginas web en código HTML y PHP. En este caso se alquiló durante un año el servicio provisto por Latinoamérica Hosting.

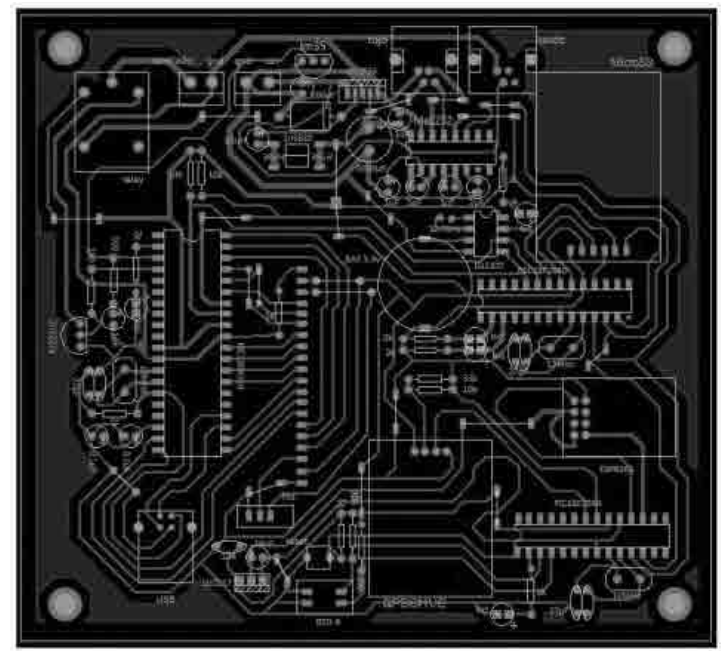

\subsection{Calibración del sistema}

Luego de ensamblar el sistema, y debido a la especificidad de los sensores utilizados, se empleó para su calibración una estación meteorológica comercial modelo WH3081 (Meteostar, 2016). La estación comercial mide los mismos parámetros del sistema meteorológico desarrollado, lo cual permite comparar las mediciones de las variables climáticas tomadas por el sistema.

La estación Meteostar se encuentra en el Instituto Rural ISER, de Pamplona, ubicada en el último piso del edificio administrativo, con una altura sobre el suelo de aproximadamente $10 \mathrm{~m}$. El sistema propuesto se ubicó en el mismo sitio para establecer la comparación.

\section{Resultados y discusión}

\subsection{Estación desarrollada}

En este apartado se describe la estación desarrollada tanto en Hardware como en software. En el hardware se incluye el circuito encargado de recibir, almacenar y dar formato a la información de las variables climáticas. En la figura 2 se visualiza en 3D la estructura del datalogger.

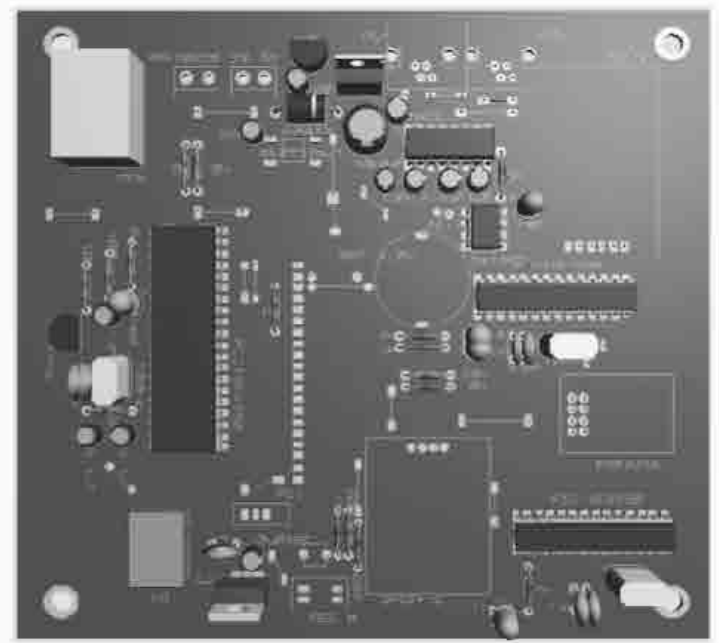

Figura 2. Diseño en proteus del datalogger. 
En cuanto al software, se diseñó un programa con ayuda de Visual Basic, para que el usuario pueda visualizar la información detallada en gráficos y registrar los datos en tiempo real. Los datos de las variables climáticas se transmiten de forma inalámbrica. También se diseñó una página WEB, a partir de los lenguajes HTML y PHP, como se muestra en la figura 3.

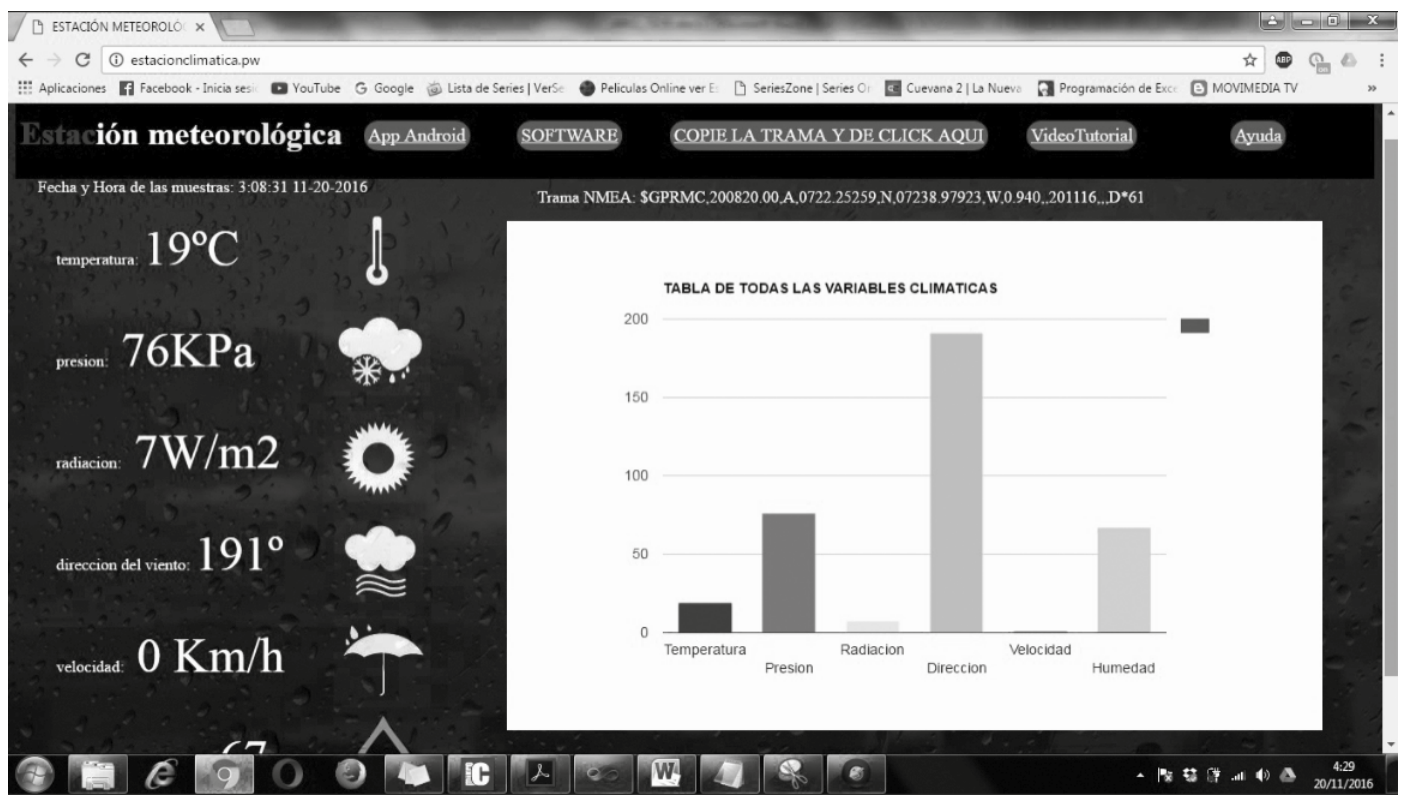

Figura 3. Página WEB de la estación meteorológica.

En la página WEB se pueden observar las seis variables, ya sea su valor numérico, o su magnitud mediante una gráfica. En la parte superior de la figura 3 se aprecian 5 pestañas, una de las cuales le brinda al usuario la opción de descargar una aplicación android para el celular.

Además, para complementar el monitoreo del sistema, se diseñó una aplicación en Android Studio, la cual tiene como función mostrarle al usuario el estado de las seis variables, junto con la trama
NMEA del GPS. El resultado final es una interfaz sencilla y cómoda, agradable al usuario, donde solo se observa la información meteorológica.

\subsection{Prueba y puesta a punto}

En la figura 4 se observa la estación meteorológica patrón, junto al sistema propuesto. Las medidas de los dos sistemas se compararon, arrojando resultados satisfactorios pues la estación desarrollada tuvo un comportamiento similar a la comercial. 


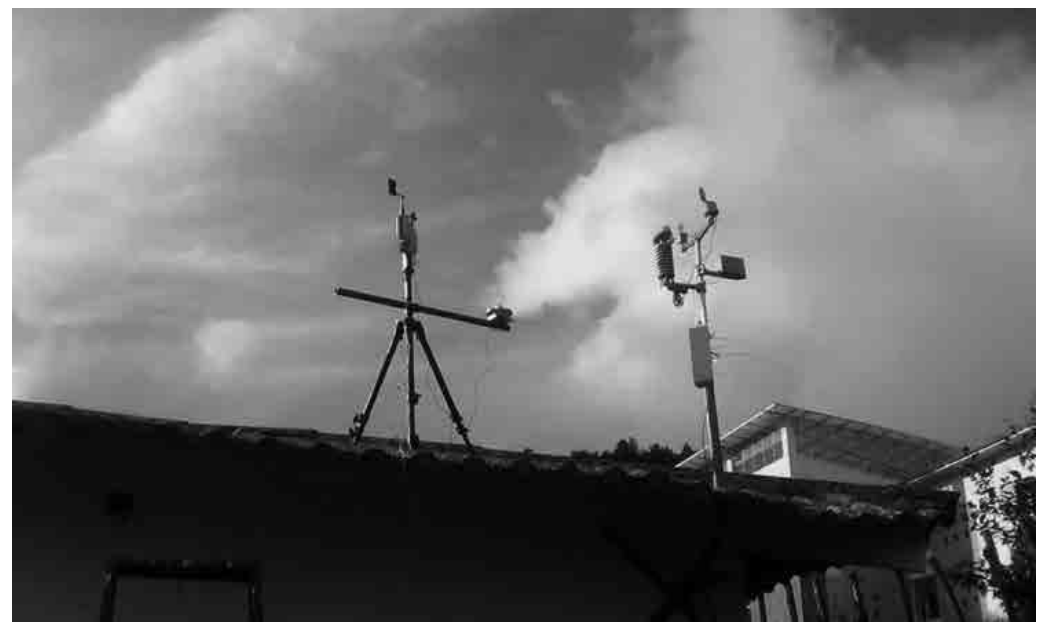

Figura 4. Fotografía de la estación patrón junto al sistema propuesto.

En las figuras 5 y 6 se observa el comportamiento de la presión atmosférica y la temperatura en las dos estaciones. Se observa que el porcentaje de error es muy pequeño, dando una precisión muy alta en las medidas.

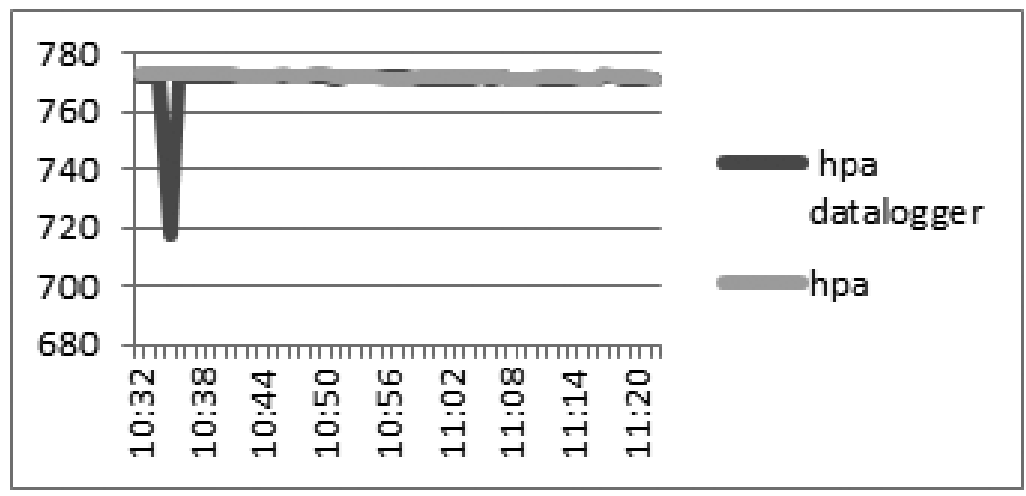

Figura 5. Presión atmosférica del sensor del sistema y el sensor patrón.

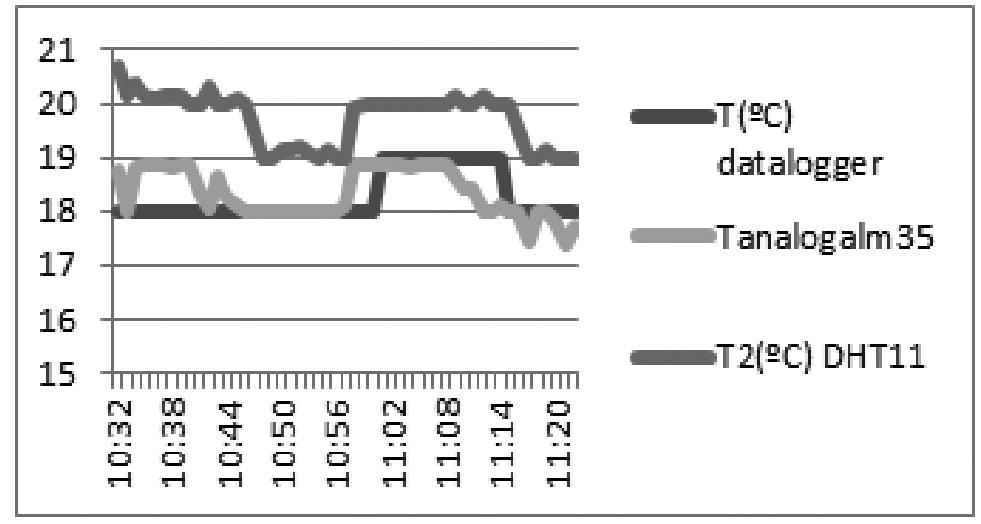

Figura 6. Gráfica de las variables de temperatura del sistema y la estación patrón del ISER. 
La puesta a punto de la estación llevada a cabo en la ciudad de Pamplona, Norte de Santander, permitió identificar que las variables climáticas más relevantes en la ciudad son la temperatura y la humedad. Esto se corroboró con los datos arrojados por la estación patrón, que se emplea en el área de agronomía.

\section{Conclusiones}

Aunque existen estaciones meteorológicas con el rótulo de automático y autónomo, no quiere decir que realicen todo un proceso de pronóstico del tiempo. Estos sistemas solo se ocupan de facilitar el proceso de medición de las variables climáticas, para que los profesionales se encarguen de la interpretación de la información.

Se diseñó el hardware del sistema, empleando componentes que se adaptaran a las necesidades que suple una estación meteorológica, como la de generar registros, almacenar información, transmitirla y visualizarla de la manera más óptima y práctica. La estación implementa un sistema de comunicación para adquisición de información proveniente de sensores de manera inalámbrica, usando un módulo WiFi y el protocolo TCP/IP. Esta característica es una ventaja para el sistema ya que facilita su portabilidad, a la vez que permite la comunicación con computadores y dispositivos móviles.

La interfaz del sistema de monitoreo, generada con Visual Basic, permite la manipulación de la estación por parte de los usuarios. Igualmente, la interfaz controla la comunicación local y externa del sistema, ya que a través de ella se envían los datos al Hosting que aloja la base de datos y la página WEB.

Al Integrar y validar el Hardware y software del sistema de monitoreo, se tuvo en cuenta equipos comerciales con tecnología actual, como estaciones digitales que cuentan con sensores calibrados, software funcionando e información que se procesa para profesionales en el campo de la agronomía.
En este sentido, los resultados permiten concluir que es posible desarrollar estaciones meteorológicas de bajo costo, a partir de dispositivos electrónicos y software de uso común, con rendimiento similar al de los equipos comerciales.

\section{Referencias}

Almario-Ospino, R., Ramón-Valencia, B. A., \& Ramón-Valencia, J. A. (2011). Sistema de adquisición de datos para el monitoreo de la calidad del agua a través de las variables de $\mathrm{pH}$, conductividad, temperatura y oxígeno disuelto. Revista Colombiana de Tecnologías de Avanzada, 1 (17), 74-80.

Altamirano-Santillán, E., Vallejo-Vallejo, G., \& Cruz-Hurtado, J. (2017). Monitoreo volcánico usando plataformas Arduino y Simulink. Revista de Investigación, Desarrollo e Innovación, 7 (2), 317-329. doi: https://doi.org/10.19053/20278306. v7.n2.2017.6073

Cargua, S., Cueva, M., Escobar, J., Arciniegas, S., \& Nieto-Guerrero, E. D. (2017). Recurso hídrico, clima y sistemas de información geográfica. Polo del Conocimiento, 2 (8), 436-470. Recuperado de: https:// polodelconocimiento.com/ojs/index.php/es/article/view/342/pdf

Figueroa-Cuello, A., Pardo-García, A., \& Díaz-Rodríguez, J. (2017). Sistema control supervisor de clientes con acceso remoto para sistemas solares fotovoltaicos autónomos. Revista de Investigación, Desarrollo e Innovación, 7 (2), 367-378. doi: https:// doi.org/10.19053/20278306.v7.n2.2017.6104

Instituto de Hidrología, Meteorología y Estudios Ambientales de Colombia, IDEAM. (2016). Atlas Climatológico de Colombia. Recuperado de: http:// www.ideam.gov.co/AtlasWeb/info/Textos/Departamentos/memoNORTESANTANDER.pdf

Márquez-Marín, R. (2004). Diseño de un sistema automatizado de medición y registro de variables climáticas para una estación meteorológica. (Tesis de 
pregrado). Universidad de Pamplona. Pamplona, Colombia.

Mercado-Ramos, V. H., Zapata, J., \& Ceballos, Y. F. (2015). Herramientas y buenas prácticas para el aseguramiento de calidad de software con metodologías ágiles. Revista de Investigación, Desarrollo e Innovación, 6 (1), 73-83. doi: https://doi. org/10.19053/20278306.3277

Meteostar (2016). Estación Meteorológica WH-3081 - MeteoStar. Recuperado de: http://www.meteostar.com.ar/descargas/estacion-\%20meteorologica-wh3081-meteostar.pdf

Microchip. (2017). Manual de microcontroladores Microchip. Recuperado de: http://www.microchip. com/

Moreno-Anselmi, L. A., Reyes-Ortiz, Ó. J., \& Ruíz-Acero, J. C. (2016). Evaluación del comportamiento mecánico de asfalto natural a partir de muestras a temperatura ambiente provenientes de Caquetá, Colombia. Revista de Investigación, Desarrollo e Innovación, 6 (2), 145-154. doi: http:// doi.org/10.19053/20278306.3115

Moreno-Rubio, J., Jiménez-López, A., \& Barrera-Lombana, N. (2013). El amplificador de potencia de carga sintonizada. Revista Colombiana de Tecnologías de Avanzada, 2 (22) 9-13. doi: https://doi. org/10.24054/16927257.v22.n22.2013.404

Organización Meteorológica Mundial, OMM. (2010). Guía de instrumentos y métodos de observación meteorológicos.

Pabón-Fernández, L., Díaz-Rodríguez, J., \& Pardo-García, A. (2016). Simulación del inversor multinivel de fuente común como variador de frecuencia para motores de inducción. Revista de Investigación, Desarrollo e Innovación, 7 (1), 165180. doi: https://doi.org/10.19053/20278306. v7.n1.2016.5636
Pardo-García, A., \& Castellanos-González, L. (2017). Automatización de ambientes en invernaderos simulando escenarios futuros. Revista Colombiana de Tecnologías de Avanzada, 1 (29).

Rodríguez-Jiménez, R., Agueda, B., \& Portela-lozano, A. (2004). Meteorología y Climatología. España: Fundación Española para la Ciencia y la Tecnología.

Sánchez-Dams, R. D. (2013). Estado del arte del desarrollo de sistemas embebidos desde una perspectiva integrada entre el hardware y software. Revista Colombiana de Tecnologías de Avanzada, 2(22), 98-105. doi: https://doi.org/10.24054/16927257. v22.n22.2013.416

Sandoval, G., Tobar-Molano, J., Mosquera, V. H., \& González, L. J. (2011). Pluviógrafo electrónico con transmisión de datos inalámbrica. Revista Colombiana de Tecnologías de Avanzada, 1 (18), 6773. doi: https://doi.org/10.24054/16927257.v17. n17.2011.173

Santiago, E. J, \& Sánchez-Allende, J. (2016). Diseño de un sistema multiagentes híbrido basado en aprendizaje profundo para la detección y contención de ciberataques. Revista Colombiana de Tecnologías de Avanzada, 2 (28), 115 - 123. doi: https://doi.org/10.24054/16927257.v28. $\mathrm{n} 28.2016 .2495$

Serna-Mendoza, C. A., Vélez-Rojas, O. A., \& Londoño-Pineda, A. A. (2016). Cambio climático, balance hídrico y eficiencia energética en algunas estaciones climáticas en Colombia. Revista Espacios, 37 (07). Recuperado de: http://www.revistaespacios. com/a16v37n07/16370707.html

Vargas-Guativa, J. A., López-Velásquez, J. A., \& Conde-Cárdenas, L. (2014). Sistema de instrumentación y control para tanques de almacenamiento de agua potable. Ingeniare, 10 (17). Recuperado de: http://www.unilibrebaq.edu.co/ojsinvestigacion/index.php/ingeniare/article/view/416 
Vázquez, R., Toledo, A., Mason, P., \& Canalí, J. (2005). Desarrollo de un procedimiento para construir un Datalogger de bajo presupuesto utilizando un dispositivo genérico, memorias seriales y tarjeta flash SD. Il jornadas de investigación en ingeniería del NEA y países limítrofes. Chaco, Argentina: Universidad Tecnológica Nacional en Resistencia. 